TITLE:

\title{
Blue light emission from strongly photoexcited and electron-doped SrTiO3
}

\section{$\operatorname{AUTHOR(S):~}$}

Yamada, Yasuhiro; Kanemitsu, Yoshihiko

\section{CITATION:}

Yamada, Yasuhiro ...[et al]. Blue light emission from strongly photoexcited and electrondoped SrTiO3. Journal of Applied Physics 2011, 109(10): 102410.

ISSUE DATE:

2011-05-31

URL:

http://hdl.handle.net/2433/141949

RIGHT:

(C) 2011 American Institute of Physics 


\title{
Blue light emission from strongly photoexcited and electron-doped $\mathrm{SrTiO}_{3}$
}

\author{
Yasuhiro Yamada, ${ }^{1, a)}$ and Yoshihiko Kanemitsu, ${ }^{1,2, b)}$ \\ ${ }^{1}$ Institute for Chemical Research, Kyoto University, Uji, Kyoto 611-0011, Japan \\ ${ }^{2}$ Photonics and Electronics Science and Engineering Center, Kyoto University, Kyoto 615-8510, Japan
}

(Received 24 July 2010; accepted 22 November 2010; published online 31 May 2011)

\begin{abstract}
We studied photoluminescence (PL) spectrum and dynamics of undoped and electron-doped $\mathrm{SrTiO}_{3}$. At low temperatures below $100 \mathrm{~K}$, we observed two band-edge PL peaks and a broad PL band in strongly photoexcited $\mathrm{SrTiO}_{3}$ and electron-doped $\mathrm{SrTiO}_{3}$ samples. In electron-doped samples, the peak energy of the broad PL band depends on the excitation photon energy at low temperatures: it changes from 2.5 to $2.9 \mathrm{eV}$ with the excitation photon energy. On the other hand, the PL dynamics is independent of the excitation photon energy and is explained by a simple model involving single-carrier trapping and Auger recombination. From the PL spectrum and dynamics under different excitation photon energies, the PL processes in $\mathrm{SrTiO}_{3}$ are discussed.

(C) 2011 American Institute of Physics. [doi:10.1063/1.3577613]
\end{abstract}

\section{INTRODUCTION}

Electronic and optical properties of transition-metal oxides are currently an area of active research. ${ }^{1,2}$ Among them, $\mathrm{SrTiO}_{3}$ has been of considerable interests as a key material of oxide-based electronics. $\mathrm{SrTiO}_{3}$ is a wide-gap semiconductor with a band-gap energy of $3.2 \mathrm{eV}$ and shows unique and multifunctional properties by electron doping. ${ }^{3-5}$ Moreover, the recent discoveries, two-dimensional electron gases (2DEGs) in $\mathrm{LaAlO}_{3} / \mathrm{SrTiO}_{3}$ heterointerface, ${ }^{6-10}$ electric-field induced metallic surface, ${ }^{11}$ and delta-doped $\mathrm{SrTiO}_{3},{ }^{12}$ prompt further interest in $\mathrm{SrTiO}_{3}$. The metallic 2DEG system shows unique electric and magnetic properties such as magnetoresistance and superconductivity at low temperatures. ${ }^{9-12}$

Very recently, we found a broad blue photoluminescence (PL) band at room temperature in electron-doped $\mathrm{SrTiO}_{3}$ and strongly photoexcited $\mathrm{SrTiO}_{3}{ }^{13}$ The spectral shape of the broad blue PL band in electron-doped $\mathrm{SrTiO}_{3}$ samples $\left(\mathrm{Nb}\right.$ or $\mathrm{La}$ doped $\mathrm{SrTiO}_{3}$, and $\mathrm{Ar}^{+}$-irradiated $\mathrm{SrTiO}_{3}$ ) is very similar to each other. ${ }^{13-17}$ Since the roomtemperature blue PL under high-density excitation reflects the intrinsic Auger recombination of photocarriers, studies of PL spectrum and dynamics in $\mathrm{SrTiO}_{3}$ provide a new opportunity for understanding electronic properties of $\mathrm{SrTiO}_{3}$. However, the PL spectrum in $\mathrm{SrTiO}_{3}$ at low temperatures is rather complicated, ${ }^{16}$ and the PL processes still remain unclear. To clarify unique low-temperature electronic properties, we need to reveal the origin of the PL and carrier recombination processes in $\mathrm{SrTiO}_{3}$ at low temperatures.

In this paper, we report the PL spectrum and dynamics in undoped and electron-doped $\mathrm{SrTiO}_{3}$. The electron-doped $\mathrm{SrTiO}_{3}$ samples show two PL bands at low temperatures (sharp and broad PL bands) and one broad PL band at high temperatures. The PL bands of doped samples are independent of dopant species. At low temperatures below $100 \mathrm{~K}$, the PL spectrum in electron-doped $\mathrm{SrTiO}_{3}$ depends on the exci-

\footnotetext{
${ }^{\text {a) }}$ Author to whom correspondence should be addressed. Electronic mail: yasuhiro.yamada@fx5.ecs.kyoto-u.ac.jp.

${ }^{\text {b)} E l e c t r o n i c ~ m a i l: ~ k a n e m i t u @ s c l . k y o t o-u . a c . j p . ~}$
}

tation photon energy. The spectral change occurs from the green PL band under low-energy excitation to the blue PL band under high-energy excitation. On the other hand, the decay dynamics of the broad PL band is independent of the excitation photon energy, and is well explained by a simple model involving single-carrier trapping and Auger recombination. Based on the excitation-energy dependence of the PL spectrum and dynamics, we discuss the PL processes in $\mathrm{SrTiO}_{3}$.

\section{EXPERIMENTAL}

We used undoped $\mathrm{SrTiO}_{3}$ single crystals and three types of electron-doped $\mathrm{SrTiO}_{3}$ single crystals $\left[\mathrm{SrTi}_{0.99} \mathrm{Nb}_{0.01} \mathrm{O}_{3}\right.$, $\mathrm{Sr}_{0.99} \mathrm{La}_{0.01} \mathrm{TiNbO}_{3}$ and $\mathrm{Ar}^{+}$-irradiated $\mathrm{SrTiO}_{3}$ (using $\mathrm{Ar}^{+}$ ions with $300 \mathrm{~V}$ acceleration voltage and $3 \mathrm{ml} / \mathrm{min} \mathrm{Ar-gas}$ flow for $10 \mathrm{~min}$ )]. All samples were $0.5 \mathrm{~mm}$ thick. Undoped samples were annealed under oxygen flow for $24 \mathrm{~h}$ at $700 \mathrm{~K}$ to reduce oxygen vacancies. We performed time-resolved PL measurements with a time resolution of about 40 ps using a streak camera and a monochromator. The excitation light source was an optical parametric amplifier system based on a regenerative amplified mode-locked Ti:sapphire laser with a pulse duration of $150 \mathrm{fs}$ and a repetition rate of $1 \mathrm{kHz}$. The laser spot size on the sample surface was measured carefully using the knife-edge method.

\section{RESULTS AND DISCUSSION}

Figure 1 shows time-integrated PL spectra of (a) strongly photoexcited undoped $\mathrm{SrTiO}_{3}$, (b) $\mathrm{Sr}_{0.99} \mathrm{La}_{0.01} \mathrm{TiO}_{3}$, (c) $\mathrm{SrNb}_{0.01} \mathrm{Ti}_{0.99} \mathrm{O}_{3}$, and (d) $\mathrm{Ar}^{+}$-irradiated $\mathrm{SrTiO}_{3}$ at 8 and $300 \mathrm{~K}$. The excitation photon energy was $3.49 \mathrm{eV}$. The PL intensities are normalized at their peaks. Note that all samples show three PL bands and the PL spectrum in each sample is similar to each other. The characteristics of three PL bands are summarized as follows.

At $300 \mathrm{~K}$, the PL spectra show a broad blue PL band at around $2.9 \mathrm{eV}$, regardless of dopant species. It was previously reported that a broad blue PL band is observed in strongly 


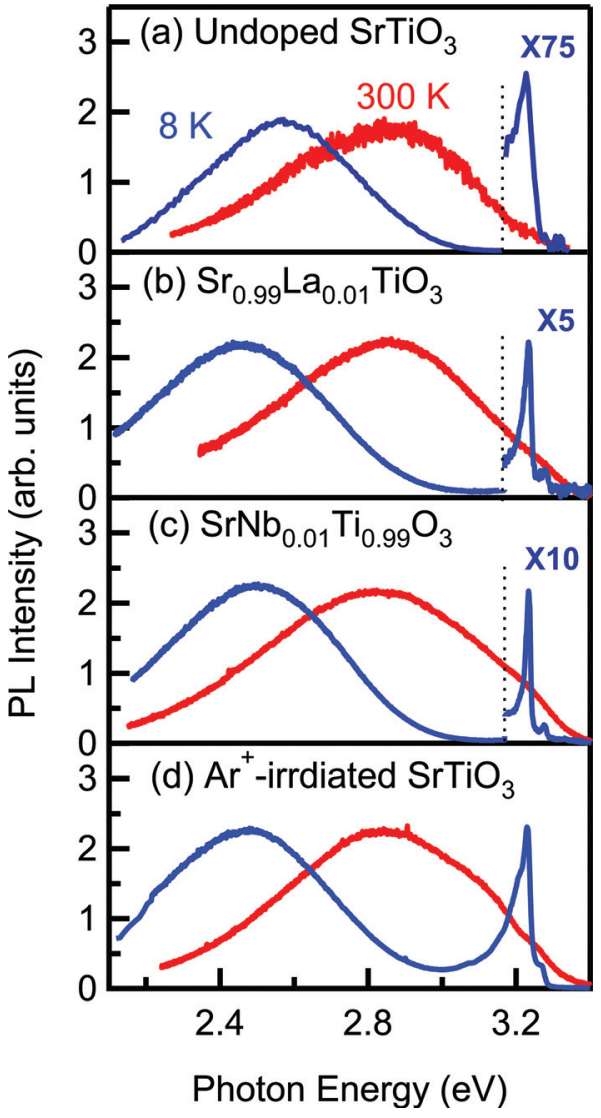

FIG. 1. (Color online) Time-integrated PL spectra of (a) strongly photoexcited undoped $\mathrm{SrTiO}_{3}$, (b) $\mathrm{Sr}_{0.99} \mathrm{La}_{0.01} \mathrm{TiO}_{3}$, (c) $\mathrm{SrNb}_{0.01} \mathrm{Ti}_{0.99} \mathrm{O}_{3}$, and (d) $\mathrm{Ar}^{+}$-irradiated $\mathrm{SrTiO}_{3}$ at 8 and $300 \mathrm{~K}$.

photoexcited $\mathrm{SrTiO}_{3}$ and electron-doped $\mathrm{SrTiO}_{3}{ }^{13,16-19}$ In addition, from the excitation-intensity dependence of the PL intensity, we clarified that the broad blue PL is caused by the bimolecular recombination of electrons and holes and the PL decay dynamics is determined by nonradiative Auger recombination. $^{13,15}$

At $8 \mathrm{~K}$, we observe sharp band-edge PL peaks at around $3.2 \mathrm{eV}$ and a green PL band at around $2.5 \mathrm{eV}$ in all $\mathrm{SrTiO}_{3}$ samples. Note that in undoped samples the $2.9 \mathrm{eV}$ broad blue PL band is also observed in the nanosecond time region by means of time-resolved PL measurement. ${ }^{16}$ Two PL peaks appear at 3.22 and $3.27 \mathrm{eV}$ in undoped samples. We have confirmed that these PL peaks coincide with the high- and low-temperature onsets of optical absorption, ${ }^{19,20}$ and concluded that the two PL peaks originate from band-to-band radiative recombination of free electrons and holes involving phonon-emission and phonon-absorption processes, where the momentum-conserving is the $25 \mathrm{meV}$ zone-edge transverse-optical phonon. ${ }^{19}$

The green PL band at around $2.5 \mathrm{eV}$ in undoped $\mathrm{SrTiO}_{3}$ shows a high quantum efficiency and a long decay time of milliseconds at low temperatures. According to the previous works, ${ }^{21-23}$ this PL origin was assigned to impurity centers or self-trapped excitons. The green PL is also observed in electron-doped $\mathrm{SrTiO}_{3}$, but its intensity is much weaker than that of undoped $\mathrm{SrTiO}_{3}$. Moreover, the PL decay of electrondoped $\mathrm{SrTiO}_{3}(\tau \sim \mathrm{ns})$ is much faster than that in undoped $\mathrm{SrTiO}_{3}$. The reduction of the PL intensity and the PL lifetime implies the importance of the impurities and defects rather than of the intrinsic self-trapped excitons.

To obtain a deeper insight on the PL processes in $\mathrm{SrTiO}_{3}$, we studied excitation-energy dependence of PL spectra at different temperatures. In undoped $\mathrm{SrTiO}_{3}$, the low-temperature PL consists of the blue and green PL bands. The blue and green PL intensities show quadratic and linear dependences on excitation density, respectively, ${ }^{16}$ and the PL spectrum depends strongly on the excitation density. On the other hand, in electron-doped $\mathrm{SrTiO}_{3}$, the PL spectrum shape is independent of the excitation density. Here, we discuss the excitation-energy dependence of the PL spectrum under weak photoexcitation using electron-doped samples.

Figure 2 shows the PL spectrum of $\mathrm{SrNb}_{0.01} \mathrm{Ti}_{0.99} \mathrm{O}_{3}$ at (a) $300 \mathrm{~K}$ and (b) $8 \mathrm{~K}$ under 3.44 and $4.00 \mathrm{eV}$ excitation. The excitation density was below $0.2 \mathrm{~mJ} / \mathrm{cm}^{2}$. At $300 \mathrm{~K}$, the PL spectrum is independent of the excitation photon energy. In contrast, at $8 \mathrm{~K}$, the PL spectra under excitation at 3.44 and $4.00 \mathrm{eV}$ are quite different. In electron-doped $\mathrm{SrTiO}_{3}$, the PL spectrum shape is independent of the excitation density but depends on the excitation photon energy under low excitation density, where the photogenerated carrier density is much less than doped carrier density.

The PL peak energy at different temperatures in $\mathrm{SrNb}_{0.01} \mathrm{Ti}_{0.99} \mathrm{O}_{3}$ is plotted as a function of the excitation photon energy in Fig. 3. At high temperatures above $200 \mathrm{~K}$, the PL peak energy is located at around $2.9 \mathrm{eV}$ and independent of the excitation photon energy. On the other hand, at low temperatures below $100 \mathrm{~K}$, the peak energy is sensitive to the excitation energy and it changes from 2.5 to 2.9 $\mathrm{eV}$ with an increase of the excitation energy. This PL spectral change indicates that the PL spectrum depends on the penetration depth of the excitation laser light.

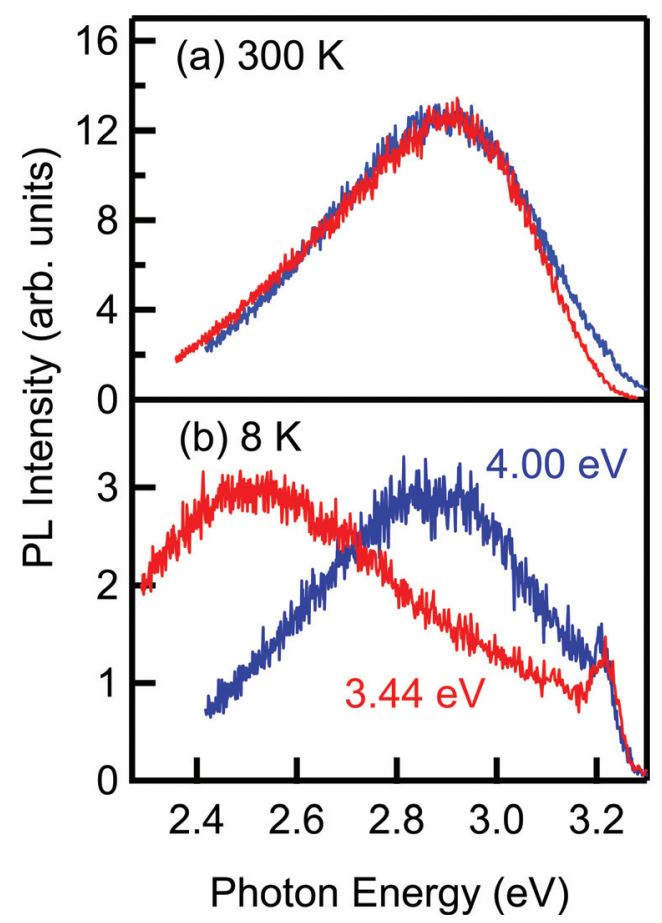

FIG. 2. (Color online) Time-integrated PL spectra of $\mathrm{SrNb}_{0.01} \mathrm{Ti}_{0.99} \mathrm{O}_{3}$ excited at 3.44 and $4.00 \mathrm{eV}$ at (a) 300 and (b) $8 \mathrm{~K}$ 


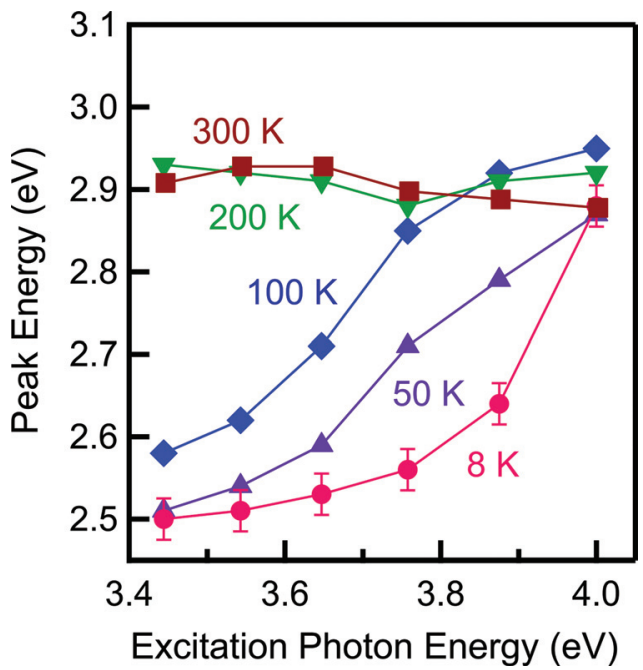

FIG. 3. (Color online) The peak energy of the broad PL band in $\mathrm{SrNb}_{0.01} \mathrm{Ti}_{0.99} \mathrm{O}_{3}$ as a function of excitation photon energy at $8,50,100$, 200 , and $300 \mathrm{~K}$

To clarify the excitation-energy dependence of the PL process, we studied the PL decay dynamics under several excitation energies. Figure 4(a) shows the PL decay dynamics of $\mathrm{SrNb}_{0.01} \mathrm{Ti}_{0.99} \mathrm{O}_{3}$ under excitation photon energies of 3.44 and $4.00 \mathrm{eV}$ at $8 \mathrm{~K}$, where the PL decay curves are monitored at the PL peak energy. The PL decay profiles show a single exponential decay and are independent of excitation photon energy, while the PL spectrum shape depends on the excitation photon energy at low temperatures (see Figs. 2 and 3).

We have previously shown that the blue PL decay dynamics of $\mathrm{SrTiO}_{3}$ is dominated by recombination of free electrons and holes. ${ }^{13,15-18}$ The carrier recombination model involves nonradiative single-carrier trapping, radiative bimolecular recombination, and nonradiative Auger recombination. This recombination model is useful in many semiconductors. ${ }^{15,16,24,25}$ In the electron-doped samples, where the doped electron density $N_{e}$ is much larger than photogenerated carrier density $n$, the carrier recombination process is dominated by the Auger recombination involving two doped electrons and a photoexcited hole. In this case $\left(N_{\mathrm{e}} \gg n\right)$, the PL dynamics is written by
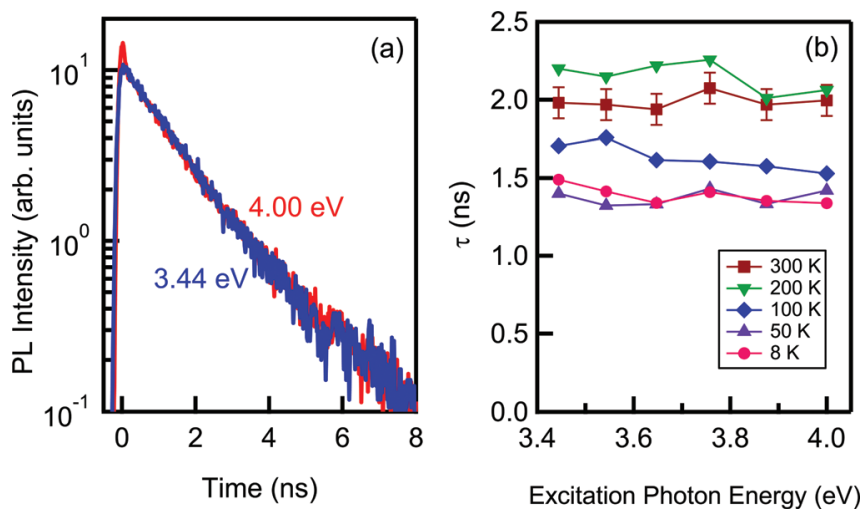

FIG. 4. (Color online) (a) PL decay dynamics of $\mathrm{SrNb}_{0.01} \mathrm{Ti}_{0.99} \mathrm{O}_{3}$ at 3.44 and $4.00 \mathrm{eV}$ at $8 \mathrm{~K}$, monitored at the PL peak energy. (b) The PL lifetime as a function of excitation photon energy at $8,50,100,200$, and $300 \mathrm{~K}$.

$$
\frac{d n}{d t}=-A n-C N_{e}^{2} n
$$

$$
I_{\mathrm{PL}} \propto B N_{e} n,
$$

where $A$ is single carrier-trapping rate. $C$ is Auger recombination coefficient involving two-doped electrons and a photoexcited hole. $B N_{e} n$ term in Eq. (2) represents the radiative bimolecular recombination. As $\mathrm{SrTiO}_{3}$ is an indirect-gap semiconductor, the bimolecular recombination rate is negligibly small (PL quantum efficiency $<1 \%$ ) compared to single carrier-trapping rate and Auger recombination rate, because wavenumber-conserving phonons are needed for radiative recombination of electrons and holes. ${ }^{16}$

According to Eqs. (1) and (2), the PL decay dynamics shows a single exponential decay with the lifetime of $\tau=\left(A+C N_{\mathrm{e}}^{2}\right)^{-1} \cdot{ }^{13,15,16}$ This is consistent with the experimental results shown in Fig. 4(a). We obtained the PL lifetime as a function of temperature by fitting the experimental data using a single exponential function. The results are summarized in Fig. 4(b). The PL lifetime is independent of the excitation photon energy in the temperature range from 8 to $300 \mathrm{~K}$. These indicate that the blue PL intensity is determined by a simple recombination process of free electrons and holes.

Finally, we discuss the PL processes in electron-doped $\mathrm{SrTiO}_{3}$. As described by Eqs. (1) and (2), the blue PL decay dynamics is dominated by nonradiative recombination of free electrons and holes. However, the blue PL origin is not the simple band-to-band recombination of free electrons and holes, as is evident from large Stokes shift of the broad blue PL band. Actually, band-to-band PL is observed at 3.22 and $3.27 \mathrm{eV} .{ }^{19}$ This fact indicates that the blue PL process involves localized states such as defects, unintentional impurities, or polaronic states. Therefore, we consider that the photoexcited free holes are localized, and immediately recombine with doped electrons for blue-light emission. ${ }^{25}$

At low temperatures, the green PL appears, and then the global PL spectrum and dynamics become complicated due to the competition between the blue and green PL processes. As shown in Figs. 3 and 4, the PL spectrum changes from green to blue as increasing the excitation photon energy, while the PL decay dynamics is unchanged. This indicates that the green and blue PL are caused by the localized-carrier recombination processes rather than free-carrier recombination processes.

The excitation-energy dependence of the PL peak energy suggests the inhomogeneous spatial distribution of deep nonradiative recombination centers for the green PL in $\mathrm{SrTiO}_{3}$ samples. ${ }^{17}$ Under high-energy excitation, the incident light is absorbed near the surface of the sample because of short penetration depth $(\sim 25 \mathrm{~nm}$ at $4.00 \mathrm{eV}$ photoexcitation, where the penetration depth is estimated from Ref. 26 using the relation of absorption coefficient $\alpha$ and penetration depth $\delta, \delta=1 / \alpha$.), and the PL under high-energy excitation reflects the electronic states only near the surface. In the near-surface region, the nonradiative recombination rate at the initial state for the green 
PL is enhanced due to a large density of deep nonradiative recombination centers. The blue PL intensity is not sensitive to the excitation photon energy, compared with the green PL intensity, indicating that the deep nonradiative recombination centers are increased by chemical doping. Therefore, the green PL is quenched in the nearsurface region, and only the blue PL is observed under high-energy photoexcitation. Under low-energy excitation, on the other hand, the green PL is observed due to the radiative recombination at deep localized states in the interior region of the samples. At high temperatures above $200 \mathrm{~K}$, the nonradiative recombination rate is much larger than the radiative recombination rate at deep states for the green PL, and thermal quenching of the green PL occurs efficiently. The excitation-energy dependence of the PL spectrum can be explained by spatial distribution of the deep nonradiative recombination centers. On the other hand, the blue PL intensity shows no significant dependence on the excitation energy, and remains at high temperatures where the green PL is quenched, as discussed above. Thus, we can conclude that the blue PL dynamics is not affected by the nonradiative recombination centers induced by impurity doping and is dominated by the intrinsic carrier recombination processes.

\section{CONCLUSION}

In conclusion, we studied PL spectrum and dynamics in strongly photoexcited $\mathrm{SrTiO}_{3}$ and electron-doped $\mathrm{SrTiO}_{3}$. The PL decay dynamics is independent of the excitation photon energy, and explained by a simple model involving Auger recombination and single-carrier trapping. The blue PL intensity is determined by recombination processes of free carriers. At low temperatures below $100 \mathrm{~K}$, we found PL spectrum changes from the blue PL under high-energy excitation to green PL under low-energy excitation. This excitation-energy dependence can be explained by the spatial distribution of deep nonradiative recombination centers. In the near-surface region, the green PL is quenched and the blue PL is only observed.

\section{ACKNOWLEDGMENTS}

The authors would like to thank Hideki Yasuda for his contribution to this work. Part of this work was supported by a Grant-in-Aid for Scientific Research on Innovative Areas "Optical Science of Dynamically Correlated Electrons" (No. 20104006) from MEXT.

${ }^{1}$ W. Eerenstein, N. D. Mathur, and J. F. Scott, Nature 442, 759 (2006).

${ }^{2}$ M. Imada, A. Fujimori, and Y. Tokura, Rev. Mod. Phys. 70, 1039 (1998).

${ }^{3}$ H. P. R. Frederikse, W. R. Thurber, and W. R. Hosler, Phys. Rev. 134, A442 (1964).

${ }^{4}$ O. N. Tufte and P. W. Chapman, Phys. Rev. 155, 796 (1967).

${ }^{5}$ J. F. Schooley, W. R. Hosler, and M. L. Cohen, Phys. Rev. Lett. 12, 474 (1964).

${ }^{6}$ A. Ohtomo and H. Y. Hwang, Nature (London) 427, 423 (2004).

${ }^{7}$ A. Tsukazaki, A. Ohtomo, T. Kita, Y. Ohno, H. Ohno, and M. Kawasaki, Science 315, 1388 (2007).

${ }^{8}$ G. Herranz, M. Basletic, M. Bibes, C. Carrétéro, E. Tafra, E. Jacquet, K. Bouzehouane, C. Deranlot, A. Hamzić, J. M. Broto, A. Barthélémy, and A. Fert, Phys. Rev. Lett. 98, 216803 (2007).

${ }^{9}$ N. Reyren, S. Thiel, A. D. Cavigla, L. Koukoutis, G. Hammerl, C. Richter, C. W. Schneider, T. Kopp, A. S. Rüetschi, D. Jaccard, M. Gabay, D. A. Muller, J. M. Triscone, and J. Mannhart, Science 317, 1196 (2007).

${ }^{10}$ S. Seri and L. Klein, Phys. Rev. B 80, 180410(R) (2009).

${ }^{11}$ K. Ueno, S. Nakamura, H. Shimotani, A. Ohtomo, N. Kimura, T. Nojima, H. Aoki, Y. Iwasa, and M. Kawasaki, Nature Mater. 7, 855 (2008).

${ }^{12}$ Y. Kozuka, M. Kim, C. Bell, B. G. Kim, Y. Hikita, and H. Y. Hwang, Nature 462, 487 (2009).

${ }^{13}$ H. Yasuda and Y. Kanemitsu, Phys. Rev. B 77, 193202 (2008).

${ }^{14}$ D. Kan, T. Terashima, R. Kanda, A. Masuno, K. Tanaka, S. Chu, H. Kan, A. Ishizumi, Y. Kanemitsu, Y. Shimakawa, and M. Takano, Nature Mater. 4, $816(2005)$

${ }^{15}$ Y. Yamada, H. Yasuda, T. Tayagaki, and Y. Kanemitsu, Appl. Phys. Lett. 95, 121112 (2009).

${ }^{16}$ Y. Yamada, H. Yasuda, T. Tayagaki, and Y. Kanemitsu, Phys. Rev. Lett. 102, 247401 (2009).

${ }^{17}$ H. Yasuda, Y. Yamada, T. Tayagaki, and Y. Kanemitsu, Phys. Rev. B 78, 233202 (2008).

${ }^{18}$ Y. Kanemitsu and Y. Yamada, Phys. Stat. Sol. (b) 248, 416 (2011).

${ }^{19}$ Y. Yamada and Y. Kanemitsu, Phys. Rev. B 82, 121103(R) (2010).

${ }^{20}$ M. Capizzi and A. Frova, Phys. Rev. Lett. 25, 1298 (1970).

${ }^{21}$ T. Hasegawa, M. Shirai, and K. Tanaka, J. Lumin. 87-89, 1217 (2000).

${ }^{22}$ R. Leonelli and J. L. Brebner, Phys. Rev. B 33, 8649 (1986).

${ }^{23}$ S. Mochizuki, F. Fujishiro, and S. Minami, J. Phys.:Condens. Mater. 17, 923 (2005).

${ }^{24} \mathrm{P}$. T. Landsberg, Recombination in Semiconductors (Cambridge University Press, Cambridge, 1991).

${ }^{25}$ Y. Yamada and Y. Kanemitsu, Phys. Rev. B 82, 113103 (2010).

${ }^{26}$ M. Cardona, Phys. Rev. 140, A651 (1965). 